\title{
Interface Landau levels in graphene monolayer-bilayer junction
}

\author{
Mikito Koshino ${ }^{1}$, Takeshi Nakanishi ${ }^{2}$, and Tsuneya Ando ${ }^{1}$ \\ ${ }^{1}$ Department of Physics, Tokyo Institute of Technology \\ 2-12-1 Ookayama, Meguro-ku, Tokyo 152-8551, Japan \\ ${ }^{2}$ Nanotube Research Center, AIST, 1-1-1 Higashi, Tsukuba 305-8565, Japan
}

(Dated: June 20, 2018)

\begin{abstract}
Electronic structure of graphene monolayer-bilayer junction in a magnetic field is studied within an effective-mass approximation. The energy spectrum is characterized by interface Landau levels, i.e., the locally flat bands appearing near the boundary region, resulting in a series of characteristic peaks in the local density of states. Their energies are independent of boundary types such as zigzag or armchair. In the atomic scale, the local density of states shows a Kekulé pattern due to the valley mixing in the armchair boundary, while does not in the zigzag boundary.
\end{abstract}

\section{INTRODUCTION}

Graphene $e^{\underline{\underline{1}} \underline{\underline{8}}}$ and its bilayer $\underline{\underline{9}} \underline{-16}$ are characterized by zero-gap band structures supporting different types of chiral particles and Landau-level structures. Recently, atomically thin graphene samples were experimentally fabricated using mechanical exfoliation ${ }^{17,18}$ and epitaxial growth,$\underline{19,20}$ The characteristic Landau-level structure and integer quantum Hall effect $t^{1,4}-\underline{7,9,13}$ were observed in magnetotransport measurements: ${ }^{21,22}$ In this paper we study the electronic structure of a hybrid system composed of monolayer and bilayer graphenes in magnetic fields.

The band structure of monolayer graphene is characterized by Dirac-like spectrum in which conduction and valence bands with linear dispersion stick at the $K$ and $K^{\prime}$ points located at a Brillouin zone corner, $\stackrel{1-3,8}{-}$ which are called valleys. Bilayer graphene has a zero-gap structure, but with quadratic dispersion unlike monolayer $\stackrel{\underline{9}}{\underline{-16}}$ In a magnetic field, the level structure of monolayer $1.4-\underline{\underline{7}}$ and bilayer $9,15,23,24$ differs in number of degeneracy at zero-energy and the quantum Hall plateaus appear at different filling factors accordingly $15,21,22$

The electronic states of graphene with an edge have been studied in theories $\underline{25}-\underline{45}$ In particular, when the boundary is along zigzag direction, special states localized at the edge appear as zero-energy modes $\stackrel{25,26}{2}$ Similar zero-energy edge states exist also in bilayer graphene. $\stackrel{46,47}{, 4}$ In a magnetic field, electron-like and hole-like Landau levels are shifted upwards and downwards near the boundary, respectively, forming edge channels away from zero energy $\stackrel{15,29,48-50}{-50}$ Recently, the transport through quantum structures consisting of monolayer and bilayer graphenes was investigated $\stackrel{51,52}{=}$ In a previous paper, the boundary condition between monolayer and bilayer graphenes connected by a monoatomic step was studied, and the transmission probability through the junction was calculated in the absence of magnetic field ${ }^{53}$

In this paper, we study the energy spectrum and local density of states of the monolayer-bilayer graphene junction in magnetic fields. Based on the previous study,$\underline{53}$ we consider a composed system of half-infinite graphene monolayer and bilayer connected by a mono- atomic step along zigzag or armchair direction. In Sec. III we present effective mass description for monolayer and bilayer graphenes and introduce formulation to describe Landau levels of the junction in Sec. III. In Sec. IV, we numerically calculate the energy spectra for several types of the boundaries as well as the local density of states. The conclusion is presented in Sec. $\mathrm{V}$

\section{EFFECTIVE MASS HAMILTONIAN}

\section{A. Monolayer graphene}

Graphene is composed of a honeycomb network of carbon atoms, containing a pair of sublattices, denoted by $A$ and $B$. Electronic states in the vicinity of $K$ and $K^{\prime}$ points in the Brillouin zone are well described by envelope functions $\left(F_{A}^{K}, F_{B}^{K}\right)$ and $\left(F_{A}^{K^{\prime}}, F_{B}^{K^{\prime}}\right)$, respectively, in an effective-mass approximation. At the $\mathrm{K}$ point, the effective Hamiltonian for $\left(F_{A}^{K}, F_{B}^{K}\right)$ is $\underline{\underline{1}} \underline{\underline{3}, \underline{8}}$

$$
\mathcal{H}^{K}=\left(\begin{array}{cc}
0 & v \pi_{-} \\
v \pi_{+} & 0
\end{array}\right),
$$

where $v \approx 1 \times 10^{6} \mathrm{~m} / \mathrm{s}$ is the band velocity $\pi_{ \pm}=\pi_{x} \pm i \pi_{y}$, and $\boldsymbol{\pi}=-i \hbar \boldsymbol{\nabla}+(e / c) \mathbf{A}$ with vector potential in the Landau gauge, $\mathbf{A}=(0, B x)$, giving external magnetic field $\mathbf{B}=\boldsymbol{\nabla} \times \mathbf{A}$. The Hamiltonian at the $K^{\prime}$ point is obtained by exchanging $\pi_{ \pm}$in Eq. (10).

The wavenumber $k_{y}$ remains a good quantum number in the present geometry. The operator $\pi_{ \pm}$can be expressed as

$$
\begin{aligned}
& v \pi_{+}=i \hbar \omega_{B} a^{\dagger}, \\
& v \pi_{-}=-i \hbar \omega_{B} a,
\end{aligned}
$$

where $\hbar \omega_{B}=\sqrt{2} \hbar v / l_{B}$ with magnetic length $l_{B}=$ $\sqrt{c \hbar /(e B)}$ and $a^{\dagger}$ and $a$ are raising and lowering operators, respectively, defined by

$$
a=\frac{\partial}{\partial z}+\frac{z}{2}
$$

with dimensionless coordinate,

$$
z=\sqrt{2}\left(\frac{x}{l_{B}}+k_{y} l_{B}\right)=\frac{\sqrt{2}(x-X)}{l_{B}} .
$$


Here, the center coordinate of the cyclotron motion is defined by

$$
X=-k_{y} l_{B}^{2}
$$

The Schrödinger equation then becomes

$$
\begin{aligned}
& \varepsilon F_{A}^{K}=-i \hbar \omega_{B} a F_{B}^{K} \\
& \varepsilon F_{B}^{K}=i \hbar \omega_{B} a^{\dagger} F_{A}^{K},
\end{aligned}
$$

giving

$$
\left(\nu-a^{\dagger} a\right) F_{B}^{K}=\left(\frac{\partial^{2}}{\partial z^{2}}+\nu+\frac{1}{2}-\frac{z^{2}}{4}\right) F_{B}^{K}=0,
$$

with

$$
\nu=\left(\frac{\varepsilon}{\hbar \omega_{B}}\right)^{2} .
$$

The independent solutions of Eq. (7) are given by $D_{\nu}(z)$ and $D_{-\nu-1}(-i z)$, where $D_{\nu}(z)$ is Weber's parabolic cylinder function defined by

$$
\begin{aligned}
D_{\nu}(z)= & 2^{\nu / 2} \sqrt{\pi} e^{-z^{2} / 4}\left[\frac{1}{\Gamma((1-\nu) / 2)} F\left(-\frac{\nu}{2}, \frac{1}{2} ; \frac{z^{2}}{2}\right)\right. \\
& \left.-\frac{\sqrt{2} z}{\Gamma(-\nu / 2)} F\left(\frac{1-\nu}{2}, \frac{3}{2} ; \frac{z^{2}}{2}\right)\right]
\end{aligned}
$$

with $F(\alpha, \gamma ; z)$ being Kummer's hypergeometric function. The components $F_{K}^{A}$ and $F_{K}^{B}$ are related by Eq. (6) with formula

$$
\begin{aligned}
a^{\dagger} D_{\nu}(z) & =D_{\nu+1}(z), \\
a D_{\nu}(z) & =\nu D_{\nu-1}(z) .
\end{aligned}
$$

Because of relation

$$
D_{\nu}(-z)=e^{\nu \pi i} D_{\nu}(z)+\frac{\sqrt{2 \pi}}{\Gamma(-\nu)} e^{(\nu+1) \pi i / 2} D_{-\nu-1}(-i z),
$$

$D_{\nu}(z)$ and $D_{\nu}(-z)$ can also be chosen as independent solutions of Eq. (7), as long as $1 / \Gamma(-\nu)$ is nonzero, i.e., $\nu$ is not 0 or a positive integer. Then, $D_{\nu}(z)$ and $D_{\nu}(-z)$ exponentially diverge in limits $z=-\infty$ and $+\infty$, respectively, while converge to zero in the opposite side. They can never be a bulk eigenfunction, but may appear when the system is half-infinite in the $x$ direction. For a nonnegative integer $n, D_{n}(z)$ and $D_{n}(-z)$ are linearly dependent, and coincide with usual Landau-level function except for a normalization factor as

$$
D_{n}(z)=(-1)^{n} D_{n}(-z)=2^{-n / 2} e^{-z^{2} / 4} H_{n}(z / \sqrt{2}),
$$

with Hermite polynomial $H_{n}(z)$. The other solution $D_{-n-1}(i z)$ then diverges both in limits $z=+\infty$ and $-\infty$ and is excluded. $D_{n}(z)$ at a negative integer $n$ generally diverges for $z \rightarrow-\infty$. At $n=-1$, for example, we have

$$
D_{-1}(z)=\sqrt{2 \pi} e^{z^{2} / 4}[-1+\operatorname{erf}(z / \sqrt{2})],
$$

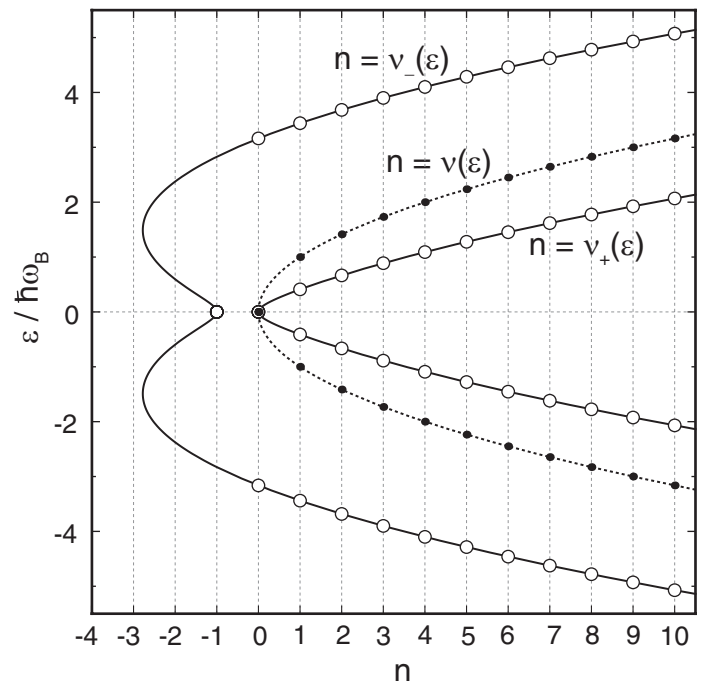

FIG. 1: Plots of $\nu(\varepsilon), \nu_{+}(\varepsilon)$, and $\nu_{-}(\varepsilon)$ with energy $\varepsilon$ set to the vertical axis. Black and white circles represent the Landau levels of bulk monolayer and bilayer, respectively. $\gamma_{1} / \hbar \omega_{B}=3$ is taken for bilayer.

with error function

$$
\operatorname{erf}(x)=\int_{0}^{x} e^{t^{2}} d t
$$

Let us define

$$
\begin{aligned}
& \phi_{\nu}^{R}(z)=D_{\nu}(z), \\
& \phi_{\nu}^{L}(z)=D_{\nu}(-z),
\end{aligned}
$$

where $L$ and $R$ represent the solutions finite in limits $z \rightarrow$ $-\infty$ and $+\infty$, respectively. We will consider a monolayerbilayer junction in which the region $x<0$ is monolayer and $x>0$ is bilayer. The eigen function in monolayer is given by

$$
\begin{aligned}
& \left(\begin{array}{c}
F_{A}^{K} \\
F_{B}^{K}
\end{array}\right)=\left(\begin{array}{c}
i \alpha_{1} \phi_{\nu-1}^{L} \\
\alpha_{2} \phi_{\nu}^{L}
\end{array}\right) e^{-i X y / l_{B}^{2}}, \\
& \left(\begin{array}{c}
\alpha_{1} \\
\alpha_{2}
\end{array}\right)=\left(\begin{array}{c}
\varepsilon / \hbar \omega_{B} \\
1
\end{array}\right) .
\end{aligned}
$$

The wavefunction at the $K^{\prime}$ point can be obtained by $\left(F_{A}^{K^{\prime}}, F_{B}^{K^{\prime}}\right)=\left(F_{B}^{K}, F_{A}^{K}\right)$.

The Landau level energies of bulk monolayer graphene are given by the condition that the wavefunction is finite in limits $x= \pm \infty$, i.e., $\nu(\varepsilon)$ is non-negative integer $n$. We get $\underline{1} \underline{\underline{4}}-\underline{\underline{7}}$

$$
\begin{aligned}
& \varepsilon_{0}=0, \\
& \varepsilon_{n, \pm}= \pm \hbar \omega_{B} \sqrt{n} \quad(n=1,2, \cdots) .
\end{aligned}
$$

The plot of $\nu(\varepsilon)$ and bulk Landau-level energies are shown in Fig. 1.

\section{B. Bilayer graphene}

Bilayer graphene is a pair of graphene layers arranged in $\mathrm{AB}$ (Bernal) stacking and includes $A_{1}$ and $B_{1}$ atoms 
on layer 1 and $A_{2}$ and $B_{2}$ on layer 2. The layers are arranged such that sites $B_{1}$ and $A_{2}$ are directly below and above each other, which are connected by interlayer coupling $\gamma_{1} \sim 0.39 \mathrm{eV}: \underline{54}$ The effective Hamiltonian for $\left(F_{A 1}^{K}, F_{B 1}^{K}, F_{A 2}^{K}, F_{B 2}^{K}\right)$ is given by ${ }^{9-16}$

$$
\mathcal{H}^{K}=\left(\begin{array}{cccc}
0 & v \pi_{-} & 0 & 0 \\
v \pi_{+} & 0 & \gamma_{1} & 0 \\
0 & \gamma_{1} & 0 & v \pi_{-} \\
0 & 0 & v \pi_{+} & 0
\end{array}\right)
$$

The Hamiltonian at the $K^{\prime}$ point is obtained by exchanging $\pi_{ \pm}$in Eq. (19).

The eigenfunction of Eq. (19) finite in limit $x \rightarrow+\infty$ is written as

$$
\left(\begin{array}{c}
F_{A 1}^{K} \\
F_{B 1}^{K} \\
F_{A 2}^{K} \\
F_{B 2}^{K}
\end{array}\right)=\left(\begin{array}{c}
-i \beta_{1}^{\mu} \phi_{\nu_{\mu}-1}^{R} \\
\beta_{2}^{\mu} \phi_{\nu_{\mu}}^{R} \\
\beta_{3}^{\mu} \phi_{\nu_{\mu}}^{R} \\
i \beta_{4}^{\mu} \phi_{\nu_{\mu}+1}^{R}
\end{array}\right) e^{-i X y / l_{B}^{2}}
$$

with

$$
\begin{gathered}
\nu_{\mu}(\varepsilon)=-\frac{1}{2}+\tilde{\varepsilon}^{2}+\frac{\mu}{2} \sqrt{4 \tilde{\varepsilon}^{2} \tilde{\gamma}_{1}^{2}+1}, \\
\left(\begin{array}{c}
\beta_{1}^{\mu} \\
\beta_{2}^{\mu} \\
\beta_{3}^{\mu} \\
\beta_{4}^{\mu}
\end{array}\right)=\left(\begin{array}{c}
\tilde{\gamma}_{1} \nu_{\mu} \tilde{\varepsilon} /\left(\tilde{\varepsilon}^{2}-\nu_{\mu}\right) \\
\tilde{\gamma}_{1} \tilde{\varepsilon}^{2} /\left(\tilde{\varepsilon}^{2}-\nu_{\mu}\right) \\
\tilde{\varepsilon} \\
1
\end{array}\right),
\end{gathered}
$$

where $\mu= \pm$ is another degree of freedom, $\tilde{\varepsilon}=$ $\varepsilon /\left(\hbar \omega_{B}\right)$, and $\tilde{\gamma}_{1}=\gamma_{1} /\left(\hbar \omega_{B}\right)$. The wavefunction at the $K^{\prime}$ point is obtained by $\left(F_{A 1}^{K^{\prime}}, F_{B 1}^{K^{\prime}}, F_{A 2}^{K^{\prime}}, F_{B 2}^{K^{\prime}}\right)=$ $\left(F_{B 2}^{K}, F_{A 2}^{K}, F_{B 1}^{K}, F_{A 1}^{K}\right)$.

The Landau levels of bulk bilayer graphene are obtained by the condition that the wavefunction of Eq. (20) is finite in limits $x \rightarrow \pm \infty$, i.e., includes only $\phi_{n}^{R}$ of non-negative integer $n$. Allowed indexes are $\nu_{+}(\varepsilon)=$ $0,1,2, \cdots$ and $\nu_{-}(\varepsilon)=-1,0,1, \cdots$. For $\nu_{-}=-1,0$ and $\nu_{+}=0$, the wavefunction Eq. (20) appears to include $\phi_{n}^{R}$ with negative $n$, but corresponding coefficient such as $\beta_{1}^{ \pm}$vanishes. At $\varepsilon=0$, there are two energy levels for $\nu_{+}=0$ and $\nu_{-}=-1.9$ Figure 1 illustrates $\nu_{ \pm}(\varepsilon)$ and bulk Landau-level energies of bilayer graphene.

\section{MONOLAYER-BILAYER JUNCTION}

We consider a composite system of monolayer and bilayer graphenes, where the left half $(x<0)$ is monolayer and the right half $(x>0)$ is AB-stacked bilayer. We assume that one layer of the bilayer part, containing $A_{1}$ and $B_{1}$ sites, seamlessly continues to the monolayer part with $A$ and $B$ sites, while the other layer composed of $A_{2}$ and $B_{2}$ sites is sharply cut at the boundary chosen as $x=0$. In the following, we consider two kinds of zigzag boundaries, zigzag-1 (ZZ1) and zigzag-2 (ZZ2) and armchair boundary (AC) as illustrated in Fig. 2 (a), (b), and (c) 53
We assume that the system infinitely continues in the $y$ direction parallel to the boundary. The wavefunctions of monolayer and bilayer regions are required to converge in limits $x=-\infty$ and $\infty$, respectively. At given energy $\varepsilon$, they can be written for the monolayer part $(x<0)$

$$
\begin{aligned}
& \left(\begin{array}{c}
F_{A}^{K} \\
F_{B}^{K}
\end{array}\right)=A^{K}\left(\begin{array}{c}
i \alpha_{1} \phi_{\nu-1}^{L}, \\
\alpha_{2} \phi_{\nu}^{L}
\end{array}\right) e^{-i X y / l_{B}^{2}}, \\
& \left(\begin{array}{c}
F_{A}^{K^{\prime}} \\
F_{B}^{K^{\prime}}
\end{array}\right)=A^{K^{\prime}}\left(\begin{array}{c}
-i \alpha_{2} \phi_{\nu}^{L} \\
\alpha_{1} \phi_{\nu-1}^{L}
\end{array}\right) e^{-i X y / l_{B}^{2}},
\end{aligned}
$$

and for the bilayer part $(x>0)$

$$
\begin{aligned}
& \left(\begin{array}{c}
F_{A 1}^{K} \\
F_{B 1}^{K} \\
F_{A 2}^{K} \\
F_{B 2}^{K}
\end{array}\right)=\sum_{\mu= \pm} B_{\mu}^{K}\left(\begin{array}{c}
-i \beta_{1}^{\mu} \phi_{\nu_{\mu}-1}^{R} \\
\beta_{2}^{\mu} \phi_{\nu_{\mu}}^{R} \\
\beta_{3}^{\mu} \phi_{\nu_{\mu}}^{R} \\
i \beta_{4}^{\mu} \phi_{\nu_{\mu}+1}^{R}
\end{array}\right) e^{-i X y / l_{B}^{2}} \\
& \left(\begin{array}{c}
F_{A 1}^{K^{\prime}} \\
F_{B 1}^{K^{\prime}} \\
F_{A 2}^{K^{\prime}} \\
F_{B 2}^{K^{\prime}}
\end{array}\right)=\sum_{\mu= \pm} B_{\mu}^{K^{\prime}}\left(\begin{array}{c}
i \beta_{4}^{\mu} \phi_{\nu_{\mu}+1}^{R} \\
\beta_{3}^{\mu} \phi_{\nu_{\mu}}^{R} \\
\beta_{2}^{\mu} \phi_{\nu_{\mu}}^{R} \\
-i \beta_{1}^{\mu} \phi_{\nu_{\mu}-1}^{R}
\end{array}\right) e^{-i X y / l_{B}^{2}}
\end{aligned}
$$

with six unknown coefficients $A^{K}, A^{K^{\prime}}, B_{ \pm}^{K}$, and $B_{ \pm}^{K^{\prime}}$ to be determined by the specific boundary condition.

\section{A. Zigzag boundary, ZZ1}

The boundary ZZ1 is parallel to the zigzag direction of honeycomb lattice, and the front-most line of bilayer part is formed by $B_{1}$ and $A_{2}$ sites. As the zigzag boundary does not mix the wavefunctions at different valleys $K$ and $K^{\prime}$, the boundary condition is separately expressed for each valley. The conditions are ${ }^{53}$

$$
\begin{aligned}
& F_{A 1}^{v}(0, y)=F_{A}^{v}(0, y), \\
& F_{B 1}^{v}(0, y)=F_{B}^{v}(0, y), \\
& F_{B 2}^{v}(0, y)=0,
\end{aligned}
$$

for $v=K$ and $K^{\prime}$. For the $K$ point, the conditions are rewritten with use of the wavefunctions (23) and (25) as

$$
M_{\mathrm{ZZ} 1}^{K}\left(\begin{array}{c}
A^{K} \\
B_{+}^{K} \\
B_{-}^{K}
\end{array}\right)=0
$$

with

$$
M_{\mathrm{ZZ} 1}^{K} \equiv\left(\begin{array}{ccc}
\alpha_{1} \phi_{\nu-1}^{L} & \beta_{1}^{+} \phi_{\nu_{+}-1}^{R} & \beta_{1}^{-} \phi_{\nu_{-}-1}^{R} \\
-\alpha_{2} \phi_{\nu}^{L} & \beta_{2}^{+} \phi_{\nu_{+}}^{R} & \beta_{2}^{-} \phi_{\nu_{-}}^{R} \\
0 & \beta_{4}^{+} \phi_{\nu_{+}+1}^{R} & \beta_{4}^{-} \phi_{\nu_{-}+1}^{R}
\end{array}\right)
$$

where the wavefunctions such as $\phi_{\nu_{\mu}}^{R}$ represent the values at $x=0$. For each $X$, the eigen energies are obtained by 


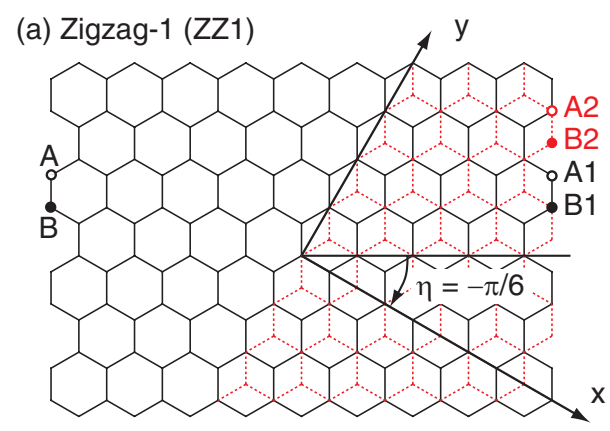

(b) Zigzag-2 (ZZ2)

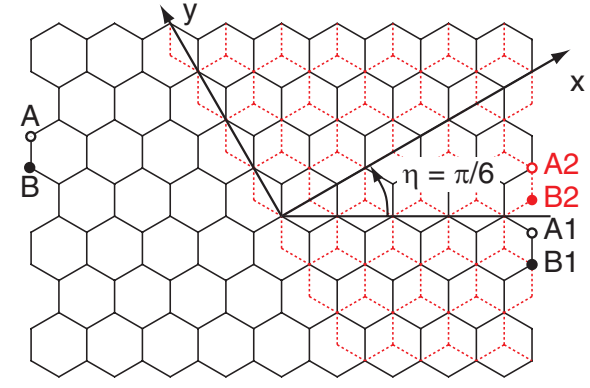

(c) Armchair (AC)

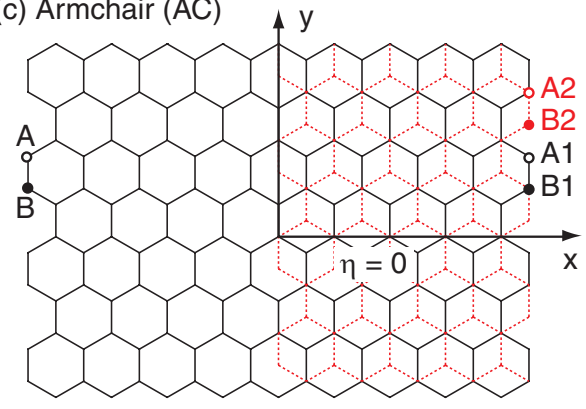

FIG. 2: Monolayer-bilayer graphene junctions with boundary types of (a) ZZ1, (b) ZZ2, and (c) AC.

searching for solutions of $\operatorname{det} M_{\mathrm{ZZ} 1}^{K}=0$. The corresponding equation for $K^{\prime}$ is

$$
\begin{gathered}
M_{\mathrm{ZZ} 1}^{K^{\prime}}\left(\begin{array}{c}
A^{K^{\prime}} \\
B_{+}^{K^{\prime}} \\
B_{-}^{K^{\prime}}
\end{array}\right)=0, \\
M_{\mathrm{ZZ} 1}^{K^{\prime}} \equiv\left(\begin{array}{ccc}
\alpha_{2} \phi_{\nu}^{L} & \beta_{4}^{+} \phi_{\nu_{+}+1}^{R} & \beta_{4}^{-} \phi_{\nu_{-}+1}^{R} \\
-\alpha_{1} \phi_{\nu-1}^{L} & \beta_{3}^{+} \phi_{\nu_{+}}^{R} & \beta_{3}^{-} \phi_{\nu_{-}}^{R} \\
0 & \beta_{1}^{+} \phi_{\nu_{+}-1}^{R} & \beta_{1}^{-} \phi_{\nu_{-}-1}^{R}
\end{array}\right) .
\end{gathered}
$$

\section{B. Zigzag boundary, ZZ2}

The boundary ZZ2 is another zigzag boundary where the front-most line of bilayer part is formed by $B_{2}$ sites. The boundary conditions are ${ }^{53}$

$$
\begin{aligned}
& F_{A 1}^{v}(0, y)=F_{A}^{v}(0, y), \\
& F_{B 1}^{v}(0, y)=F_{B}^{v}(0, y), \\
& F_{A 2}^{v}(0, y)=0,
\end{aligned}
$$

where only the third condition is different from Eq. (28). Similarly to ZZ1, we obtain the matrix for the $K$ and $K^{\prime}$ points

$$
\begin{aligned}
M_{\mathrm{ZZ} 2}^{K} & \equiv\left(\begin{array}{ccc}
\alpha_{1} \phi_{\nu-1}^{L} & \beta_{1}^{+} \phi_{\nu_{+}-1}^{R} & \beta_{1}^{-} \phi_{\nu_{-}-1}^{R} \\
-\alpha_{2} \phi_{\nu}^{L} & \beta_{2}^{+} \phi_{\nu_{+}}^{R} & \beta_{2}^{-} \phi_{\nu_{-}}^{R} \\
0 & \beta_{3}^{+} \phi_{\nu_{+}}^{R} & \beta_{3}^{-} \phi_{\nu_{-}}^{R}
\end{array}\right), \\
M_{\mathrm{ZZ} 2}^{K^{\prime}} \equiv & \left(\begin{array}{ccc}
\alpha_{2} \phi_{\nu}^{L} & \beta_{4}^{+} \phi_{\nu_{+}+1}^{R} & \beta_{4}^{-} \phi_{\nu_{-}+1}^{R} \\
-\alpha_{1} \phi_{\nu-1}^{L} & \beta_{3}^{+} \phi_{\nu_{+}}^{R} & \beta_{3}^{-} \phi_{\nu_{-}}^{R} \\
0 & \beta_{2}^{+} \phi_{\nu_{+}}^{R} & \beta_{2}^{-} \phi_{\nu_{-}}^{R}
\end{array}\right) .
\end{aligned}
$$

\section{Armchair boundary (AC)}

The boundary conditions for the armchair boundary $\mathrm{AC}$ are ${ }^{53}$

$$
\begin{aligned}
& F_{A 1}^{v}(0, y)=F_{A}^{v}(0, y), \\
& F_{B 1}^{v}(0, y)=F_{B}^{v}(0, y), \\
& F_{A 2}^{K}(0, y)-F_{A 2}^{K^{\prime}}(0, y)=0, \\
& F_{B 2}^{K}(0, y)+F_{B 2}^{K^{\prime}}(0, y)=0,
\end{aligned}
$$

where the third and fourth conditions mix the wavefunctions at the $K$ and $K^{\prime}$ points. They are rewritten as

$$
M_{\mathrm{AC}}\left(\begin{array}{c}
A^{K} \\
B_{+}^{K} \\
B_{-}^{K} \\
A^{K^{\prime}} \\
B_{+}^{K^{\prime}} \\
B_{-}^{K^{\prime}}
\end{array}\right)=0,
$$

with

$$
\begin{aligned}
M_{\mathrm{AC}} & =\left(\begin{array}{cc}
M_{\mathrm{ZZ} 1}^{K} & M^{K K^{\prime}} \\
M^{K^{\prime} K} & M_{\mathrm{ZZ} 2}^{K^{\prime}}
\end{array}\right), \\
M^{K K^{\prime}} & =\left(\begin{array}{ccc}
0 & 0 & 0 \\
0 & 0 & 0 \\
0 & -\beta_{1}^{+} \phi_{\nu_{+}-1}^{R} & -\beta_{1}^{-} \phi_{\nu_{-}-1}^{R}
\end{array}\right), \\
M^{K^{\prime} K} & =\left(\begin{array}{ccc}
0 & 0 & 0 \\
0 & 0 & 0 \\
0 & \beta_{3}^{+} \phi_{\nu_{+}}^{R} & \beta_{3}^{-} \phi_{\nu_{-}}^{R}
\end{array}\right)
\end{aligned}
$$

\section{Interface Landau levels}

Let us consider a special state of valley $v$, which satisfies the conditions

$$
\begin{aligned}
& F_{A 1}^{v}(0, y)=F_{A}^{v}(0, y), \\
& F_{B 1}^{v}(0, y)=F_{B}^{v}(0, y), \\
& F_{A 2}^{v}(0, y)=0, \\
& F_{B 2}^{v}(0, y)=0 .
\end{aligned}
$$

Because these include both boundary conditions for ZZ1 and ZZ2, such a state must be shared by both ZZ1 and ZZ2. Those states exist at different series of points $(\varepsilon, X)$ 
for $v=K$ and $K^{\prime}$, denoted by $P_{K}$ and $P_{K^{\prime}}$, respectively. Further, the wavefunction satisfying Eq. (40) at valley $v$ also meets conditions (35) for the armchair boundary, when the wave amplitudes of the other valley (opposite valley of $v$ ) are all zero. As a result, points $P_{K}$ and $P_{K^{\prime}}$ are also shared by Landau levels in an armchair boundary.

Using some algebra, we can show that at the special points $P_{K}$ and $P_{K^{\prime}}$, the gradient of the Landau level energy in $X$ vanishes in any types of boundaries ZZ1, ZZ2, and $\mathrm{AC}$. We can show that the second derivative also vanishes for ZZ2. The detailed proof is presented in Appendix A Accordingly the density of states diverges at the identical energies independent of the boundary type. Further, at those points, the wavefunctions of monolayer part and bilayer part connect smoothly on layer 1, because the amplitude on layer 2 locally vanishes and thus hardly affects the electron motion on layer 1 . As a result, the wavefunctions on the monolayer and bilayer sides are coupled well, and the amplitude is almost equally distributed to both sides.

As will be demonstrated in numerical results presented in the next section, in ZZ1 and $\mathrm{AC}, \varepsilon(X)$ takes a local maximum at each $P_{K}$ and $P_{K^{\prime}}$ in positive energies, while there usually exists another point nearby where $\varepsilon(X)$ takes a local minimum, giving divergent density of states as well. We will show that, around these points, a crossover takes place from a monolayer edge-state mainly localized in monolayer, to a bilayer edge-state mainly localized in bilayer, when $X$ is varied. It is natural that slight shift in $X$ does not change the energy at such crossover points, because they are anti-crossing points between intersecting energy levels of monolayer and bilayer edgestates. In ZZ2, the energy minima and maxima are degenerate corresponding to vanishing second derivative, and thus $\varepsilon(X)$ is even smoother and the divergence in the density of states is stronger than ZZ1 and AC. These nearly flat-band regions around extrema of $\varepsilon(X)$ can be referred to as the interface Landau levels.

\section{E. Zero energy levels}

The energy spectrum of a monolayer-bilayer junction approaches that of bulk monolayer and bilayer graphenes in the limit of $X \rightarrow+\infty$ and $-\infty$, respectively, because the wave function, centered at $x=X$, mostly resides in the bulk region far from the boundary. On the other hand, the zero-energy level is special in that it is contributed not only by the bulk Landau levels, but also by the zero-energy edge states, which are localized near the boundary region on the terminated layer of bilayer graphene .53 We can analytically obtain the energies and wavefunctions of zero energy Landau levels using the above formulation, as demonstrated in Appendix B for ZZ2 boundary. Table $\$ summarizes the degeneracy of zero energy levels in the limit of $X \rightarrow \pm \infty$ for each boundary type, where +1 represents the additional de- (a) ZZ1

(b) ZZ2

(c) $\mathrm{AC}$

\begin{tabular}{|c|c|c|c|c|c|c|c|c|}
\hline$X$ & $-\infty$ & $+\infty$ & $X$ & $-\infty$ & $+\infty$ & $X$ & $-\infty$ & $+\infty$ \\
\hline$K$ & 1 & $2+1$ & $K$ & $1+1$ & 2 & $K$ & 1 & 2 \\
\hline$K^{\prime}$ & $1+1$ & 2 & $\overline{K^{\prime}}$ & 1 & $2+1$ & $\overline{K^{\prime}}$ & 1 & 2 \\
\hline
\end{tabular}

TABLE I: Number of zero-energy Landau levels per spin in the limit of $X= \pm \infty$, for (a) zigzag-1, (b) zigzag-2, and (c) armchair boundaries. +1 represents extra degeneracy due to the zero-energy edge mode.

generacy due to the edge states. In ZZ1 and ZZ2, the edge state appears either of $X= \pm \infty$ depending on valleys, while it is absent in $\mathrm{AC}$

\section{F. Local density of states}

In monolayer graphene, the amplitude of the wavefunctions at $A$ and $B$ sites are written in terms of effectivemass envelope functions as ${ }^{8}$

$$
\begin{aligned}
& \psi_{A}(\mathbf{R})=e^{i \mathbf{K} \cdot \mathbf{R}} F_{A}^{K}(\mathbf{R})+e^{i \eta} e^{i \mathbf{K}^{\prime} \cdot \mathbf{R}} F_{A}^{K^{\prime}}(\mathbf{R}), \\
& \psi_{B}(\mathbf{R})=-\omega e^{i \eta} e^{i \mathbf{K} \cdot \mathbf{R}} F_{B}^{K}(\mathbf{R})+e^{i \mathbf{K}^{\prime} \cdot \mathbf{R}} F_{B}^{K^{\prime}}(\mathbf{R}),
\end{aligned}
$$

where $\eta$ is the angle between the $x$ axis and zigzag direction of honeycomb lattice and $\omega=e^{2 \pi i / 3}$. In bilayer graphene, the amplitude can be written as ${ }^{53}$

$$
\begin{aligned}
& \psi_{A 1}(\mathbf{R})=e^{i \mathbf{K} \cdot \mathbf{R}} F_{A 1}^{K}(\mathbf{R})+e^{i \eta} e^{i \mathbf{K}^{\prime} \cdot \mathbf{R}} F_{A 1}^{K^{\prime}}(\mathbf{R}), \\
& \psi_{B 1}(\mathbf{R})=-\omega e^{i \eta} e^{i \mathbf{K} \cdot \mathbf{R}} F_{B 1}^{K}(\mathbf{R})+e^{i \mathbf{K}^{\prime} \cdot \mathbf{R}} F_{B 1}^{K^{\prime}}(\mathbf{R}), \\
& \psi_{A 2}(\mathbf{R})=-\omega e^{i \eta} e^{i \mathbf{K} \cdot \mathbf{R}} F_{A 2}^{K}(\mathbf{R})+e^{i \mathbf{K}^{\prime} \cdot \mathbf{R}} F_{A 2}^{K^{\prime}}(\mathbf{R}), \\
& \psi_{B 2}(\mathbf{R})=\omega^{-1} e^{2 i \eta} e^{i \mathbf{K} \cdot \mathbf{R}} F_{B 2}^{K}(\mathbf{R})+e^{-i \eta} e^{i \mathbf{K}^{\prime} \cdot \mathbf{R}} F_{B 2}^{K^{\prime}}(\mathbf{R}) .
\end{aligned}
$$

In a tight-binding model, the local density of state (LDOS) on site $A$ located at the position $\mathbf{R}$ is defined by

$$
\rho_{A}(\varepsilon ; \mathbf{R})=\sum_{\alpha} \delta\left(\varepsilon-\varepsilon^{(\alpha)}\right)\left|\psi_{A}^{(\alpha)}(\mathbf{R})\right|^{2},
$$

where $\varepsilon^{(\alpha)}$ and $\psi^{(\alpha)}$ are the energy and the wavefunction of eigenstate $\alpha$. Similar expressions can be written down for other sites $B, A 1$, etc. When the wave amplitudes at the $K$ and $K^{\prime}$ points coexist in a single eigenstate, LDOS has a Kekulé pattern due to the interference between the factors $e^{i \mathbf{K} \cdot \mathbf{R}}$ and $e^{i \mathbf{K}^{\prime} \cdot \mathbf{R}} \underline{55}$ In the present case, this is expected to appear in the armchair boundary which mixes the $K$ and $K^{\prime}$ valleys, while absent in ZZ1 or ZZ2, where every eigenstate is a single-valley state. We also define the spatially averaged LDOS for site $A$ as

$$
\rho_{A}^{\mathrm{av}}(\varepsilon ; \mathbf{R})=\sum_{\alpha} \sum_{v=K, K^{\prime}} \delta\left(\varepsilon-\varepsilon^{(\alpha)}\right)\left|\left(F^{(\alpha)}\right)_{A}^{v}(\mathbf{R})\right|^{2} .
$$

This is an average of the original LDOS in Eq. (43) over several unit cells in the region smaller than typical length scales of the envelope function. 
(a) Zigzag-1

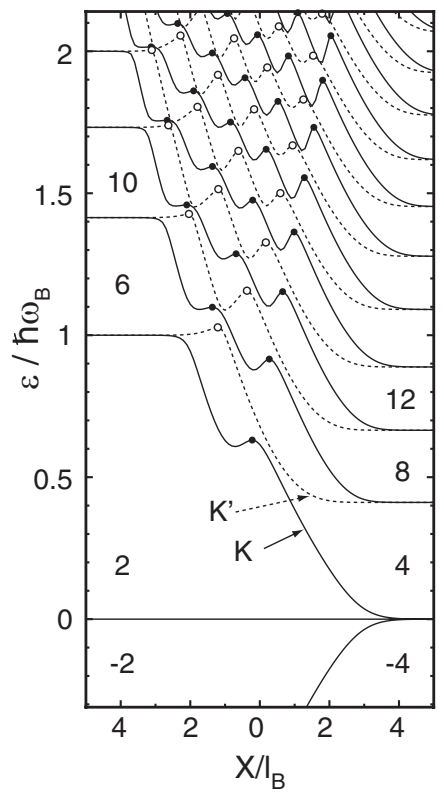

(b) Zigzag-2

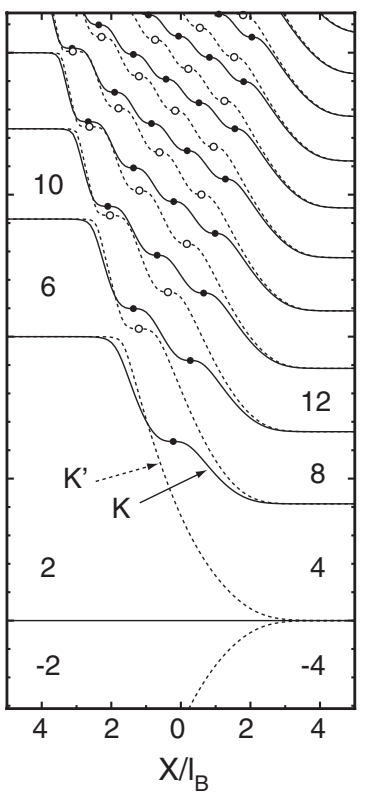

(c) Armchair

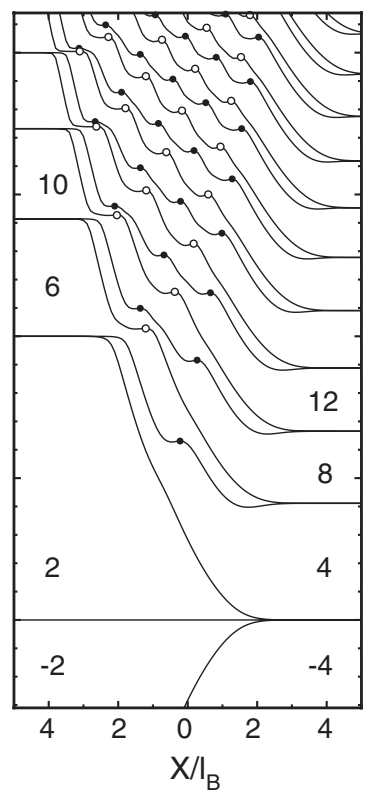

FIG. 3: Energy spectrum as a function of $X$ in boundary (a) ZZ1, (b) ZZ2, and (c) AC, at magnetic field $\hbar \omega_{B}=\gamma_{1} / 3$ $(B \sim 10 \mathrm{~T})$. Black and white circles represent $P_{K}$ and $P_{K^{\prime}}$, respectively. Numbers between the levels indicate bulk filling factor in limits $X= \pm \infty$.

\section{NUMERICAL RESULTS}

Figure 3] shows the energy spectra against $X$, numerically calculated for the junctions of ZZ1, ZZ2, and AC boundaries at magnetic field of $\hbar \omega_{B}=\gamma_{1} / 3(B \sim 10 \mathrm{~T})$. Landau levels approach those of bulk monolayer and of bilayer in the limit $X \rightarrow \infty$ and $-\infty$, respectively. In the boundary region, the valley-degenerate levels split and connect to different levels in the opposite side. The black and white circles represent the interface Landau levels $P_{K}$ and $P_{K^{\prime}}$, respectively, which are independent of boundary type and corresponds to local band maxima. In accordance with the argument in Sec. IIID we actually see that energy levels pass through those points in all three cases and the gradient vanishes there. In ZZ1 and $\mathrm{AC}$, the band minima are also present near the maxima at $P_{K}$ and $P_{K}^{\prime}$, while in ZZ2 the minima and the maxima merge into inflection points as the second derivative vanishes.

The oscillatory band structures appearing in the boundary region can be understood in relation to terminated monolayer and bilayer graphenes. Let us take ZZ1 boundary, and consider a system with infinite on-site potential added on an array of $B$ sites near the boundary, as illustrated as white circles in the top panel of Fig. 4. The system is then separated into monolayer terminated with Klein's edge and bilayer terminated with zigzag edge. In the effective mass approximation, this is equivalent to the boundary condition $F_{B}^{v}=0$ for monolayer and $F_{B 1}^{v}=F_{B 2}^{v}=0$ for bilayer. Note that in the effective-mass approximation, shifting of on-site poten- tial position by the order of the lattice constant does not make a difference in the result.

Lower panels of Fig. 4 show the energy spectrum of ZZ1 (solid lines) and that of the terminated system (dashed lines), for each of $K$ and $K^{\prime}$. In the terminated system, the independent Landau levels of monolayer and bilayer sharply go up as $X$ goes over the boundary. $\underline{29,48}$ Apparently, the spectrum of ZZ1 resembles that of the terminated system, with an energy gap opened at every crossing point. The resemblance of the two different spectra may be attributed to following reasons. In the monolayer-bilayer junction, when a low-energy electron travels from the monolayer to the bilayer, it feels as if $B$ sites suddenly disappear at the boundary, because in bilayer, $B_{1}$ is coupled with $A_{2}$ to make high-energy states away from $\varepsilon=0$. This effect can be roughly modeled by on-site potential at $B$ sites at the boundary. For an electron coming from bilayer side, on the other hand, $B_{1}$ site which was absent in the low-energy spectrum suddenly resumes at the beginning of the monolayer region, while $A_{1}$ just smoothly connects to $A$. This should roughly correspond to some condition for $B_{1}$ sites, with $A_{1}$ left intact. Energy gaps opening at crossing points are due to finite hybridization between monolayer and bilayer states.

As another remark, we observe that energy levels of ZZ1 pass through every crossing point of terminated bilayer and monolayer levels. This occurs when an eigenfunction of ZZ1 happens to have a node on the on-site potential sites, because such a state is also an eigenstate when on-site potential is present. Therefore the wavefunction of ZZ1 becomes identical with that of the ter- 
Zigzag-1
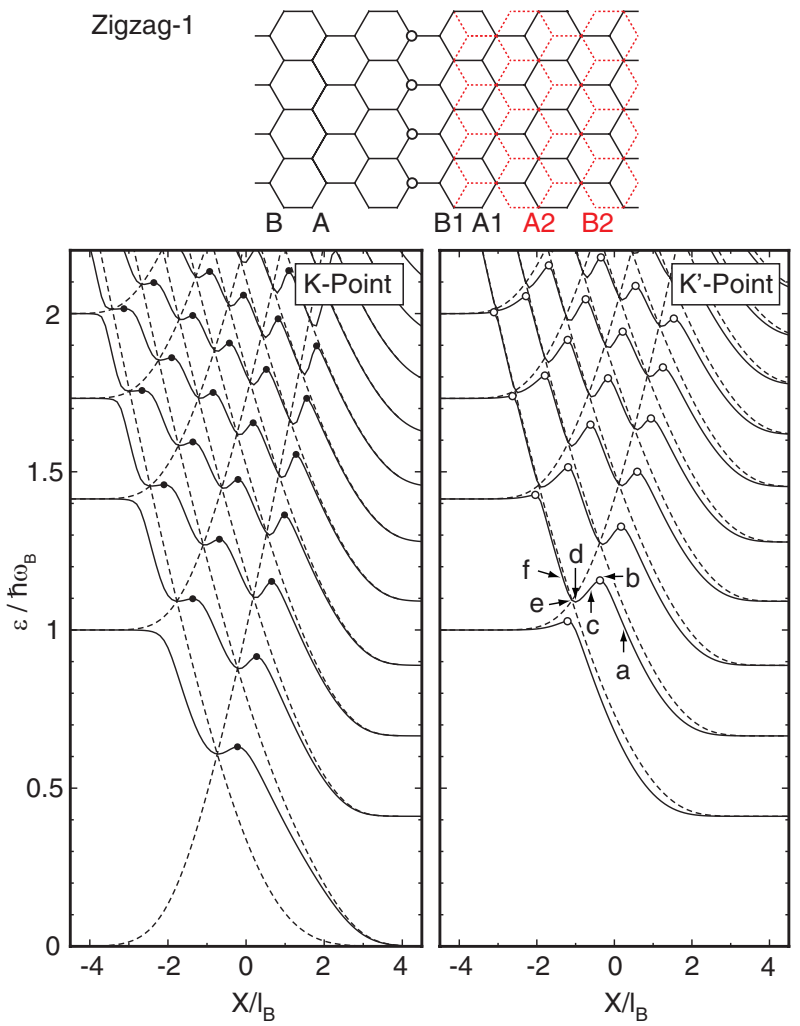

FIG. 4: (Above) Monolayer-bilayer junction of type ZZ1, separated by infinite potential on white circles into independent monolayer and bilayer graphenes. (Below) Energy spectrum of junction ZZ1 (solid) and separated system (dashed), in magnetic field $\hbar \omega_{B}=\gamma_{1} / 3$. Left and right panels show the spectra of the $K$ and $K^{\prime}$ points, respectively, and the black and white circles are the interface Landau levels.

minated system at each crossing point.

Similar analysis is also available in boundary ZZ2. Figure 5 compares the energy spectrum of ZZ2, and that of separated system with on-site potential on $B$ sites illustrated in the top panel. The boundary condition becomes $F_{B}^{v}=0$ for monolayer and $F_{B 1}^{v}=F_{A 2}^{v}=0$ for bilayer. Since the low-energy spectrum of the bilayer is dominated by $A 1$ and $B 2$, the second condition $F_{A 2}^{v}=0$ should give a weaker effect compared to $F_{B 2}^{v}=0$ in ZZ1 and thus leads to better coupling between the monolayer and bilayer region. In Fig. [5] indeed, the mixing between terminated levels looks stronger than in ZZ1, resulting in the monotonic dependence rather than non-monotonic behavior in ZZ1.

Figure 6 plots the wavefunctions near an interface Landau levels associated with the $K^{\prime}$ point in ZZ1, where (a) to (f) correspond to the points in the energy spectrum in Fig. 4. The point (b) is exactly at a local maximum $P_{K^{\prime}}$. There, the wavefunction of layer 1 smoothly connects at the boundary as argued in Sec. IIID, while generally not in other cases. The point (e) is exactly at the crossing point of terminated levels. There, the wave function indeed has a node at the interface for the $B$ and $B 1$
Zigzag-2
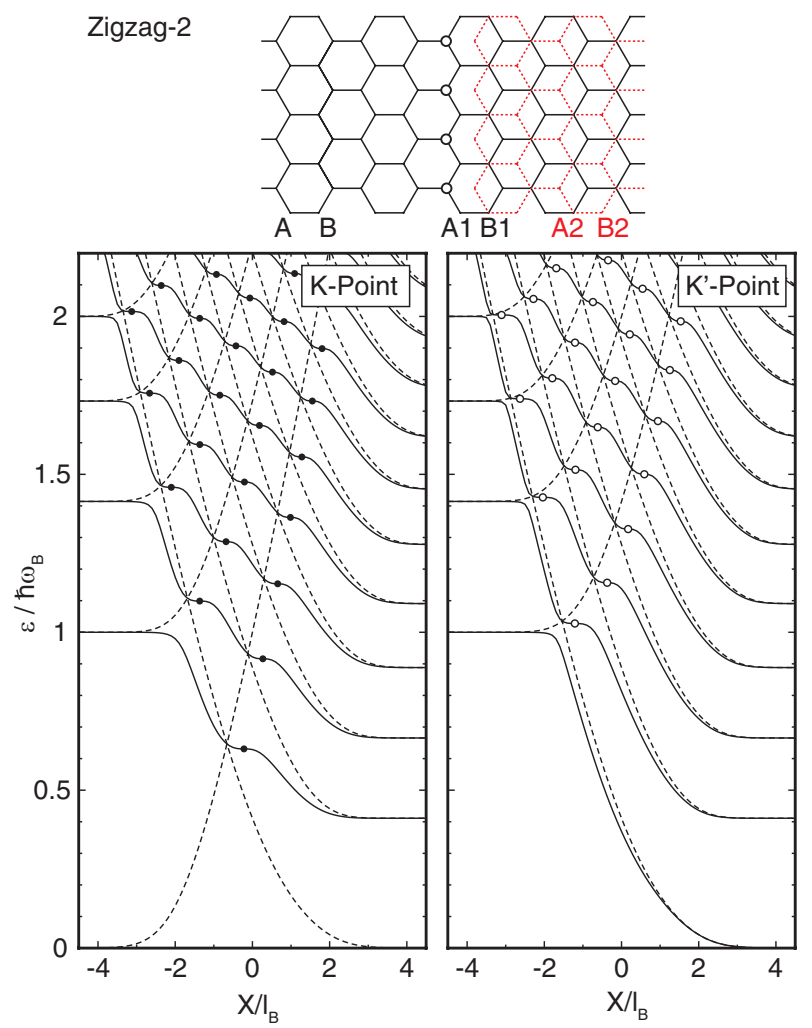

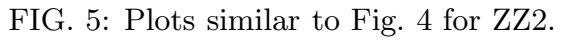

components and thus can be an eigenstate of the separate monolayer and bilayer. At the local band minimum (d), the wave function does not have special features in contrast to $P_{K^{\prime}}$.

In the energy spectrum, the region between (a) and (b) and between (e) and (f) have slope close to that of the terminated bilayer, while the region between (b) and (d) has slope close to that of the terminated monolayer. Correspondingly, the wavefunctions of (a) and (f) has significant amplitudes in bilayer side, while (c) has great amplitude in monolayer side.

Figure 7 illustrates the typical atomic-scale LDOS of Eq. (43) at interface Landau levels. We here take the boundary ZZ2 at the energy of the lowest interface Landau level near $\varepsilon=0.631 \hbar \omega_{B}$ in Fig. 3(b). The ratio of the magnetic length to the lattice constant, $l_{B} / a$, is about 30 at this magnetic field. The areas of circles in upper and lower panels represent the relative amplitude of LDOS at each atom on layers 1 and 2, respectively, while open and filled circles represent the A and B sublattices. The result mainly reflects the wavefunction of interface Landau level, since the flat band gives a dominant contribution to LDOS. We see that the wave amplitude on layer 1 connects smoothly at the boundary region, as the amplitude of layer 2 is almost absent there.

For comparison, we show the similar plot of LDOS of the armchair boundary at a different energy $\varepsilon=0.3 \hbar \omega_{B}$ in Fig. 8. In accordance with the previous argument, the plot clearly exhibits the Kekulé pattern unlike in ZZ2. 


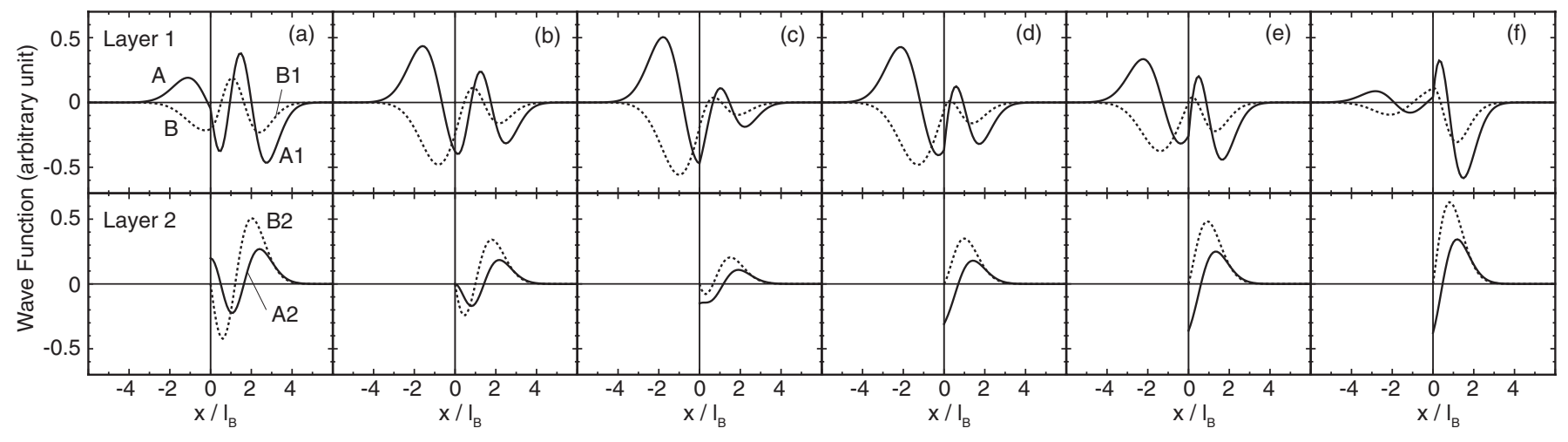

FIG. 6: Wavefunctions near the interface Landau level of the $K^{\prime}$ point in ZZ1 at $\hbar \omega_{B}=\gamma_{1} / 3$. (a) to (f) correspond to the points in the energy spectrum in Fig. 4.
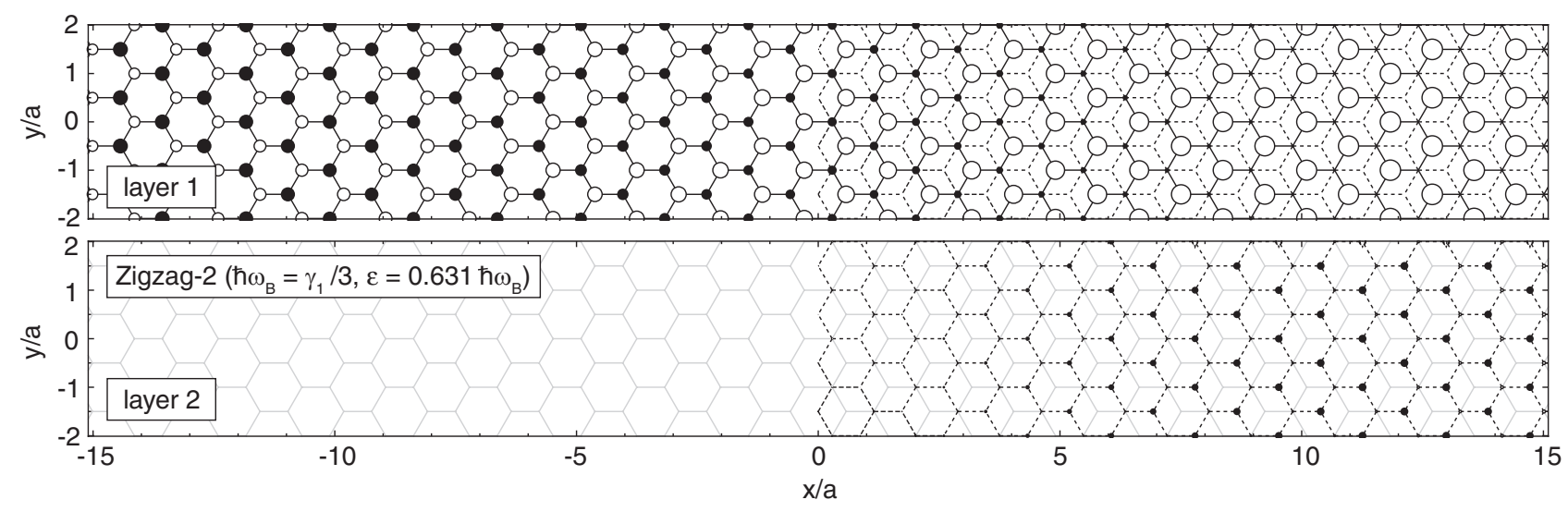

FIG. 7: Local density of states of boundary ZZ2 with $\hbar \omega_{B}=\gamma_{1} / 3$, at the energy $\varepsilon=0.631 \hbar \omega_{B}$ near the lowest interface Landau level of $K$. The areas of circles in upper and lower panels represent the amplitude of LDOS at each atom on the layer 1 and 2, respectively, while open and filled circles represent $A$ and $B$ sublattices, respectively. $l_{B} / a$ is about 30 at this magnetic field.

Note that, even in the armchair boundary, the Kekulé pattern disappears when the energy comes to an interface Landau level, because the eigenfunction becomes a singlevalley state there.

Figure 9 shows the averaged local density of states (LDOS) defined in Eq. (44), for ZZ2 boundary. The vertical scale is shared with the corresponding energy spectrum at left. We observe series of peaks corresponding to the interface Landau levels of $P_{K}$ and $P_{K^{\prime}}$ owing to the large LDOS due to the flat band, and its spatial distribution is characterized by node pattern of the corresponding wavefunction. While not shown, the peak patterns are quite similar among ZZ1, ZZ2, and AC, since every interface Landau level appears at the identical energy with the identical effective-mass wavefunction. In ZZ1 and $\mathrm{AC}$, the band minima appearing near $P_{K}$ and $P_{K^{\prime}}$ also contribute to the LDOS divergence and the peak structure is a little blurred.

Near the interface Landau levels, LDOS has a considerable amplitude in monolayer region, while otherwise it is localized mostly in the bilayer region. This is because the monolayer and bilayer states are well hybridized near the interface Landau levels, while in other regions where the band lines are downslope, the states mainly originate from bilayer, as argued above.

\section{CONCLUSION}

We have studied electronic structures of monolayerbilayer graphene junctions in magnetic fields. The energy spectrum near the boundary region is characterized by the interface Landau levels where the band energy is locally constant, which arise from hybridization of Landau levels of terminated monolayer and bilayer graphenes. The energies of interface Landau levels are insensitive to the way the second layer is terminated, suggesting that they would be robust even in a disordered junction containing a random atomic configuration at the boundary. Interface Landau levels give a characteristic peak pattern to LDOS, which may be observed by scanning spectroscopic measurement $\underline{\underline{56} \underline{\underline{59}}}$ 


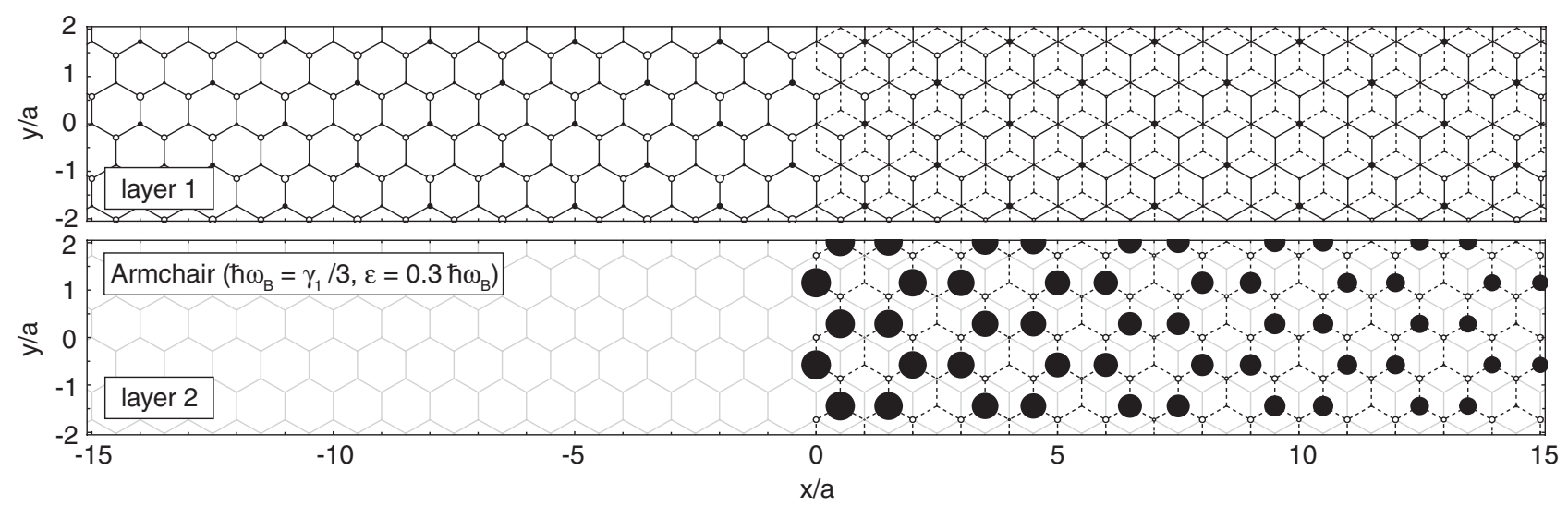

FIG. 8: Plot similar to Fig. 7 showing the local density of states of the armchair boundary with $\hbar \omega_{B}=\gamma_{1} / 3$ at the energy $\varepsilon=0.3 \hbar \omega_{B}$.
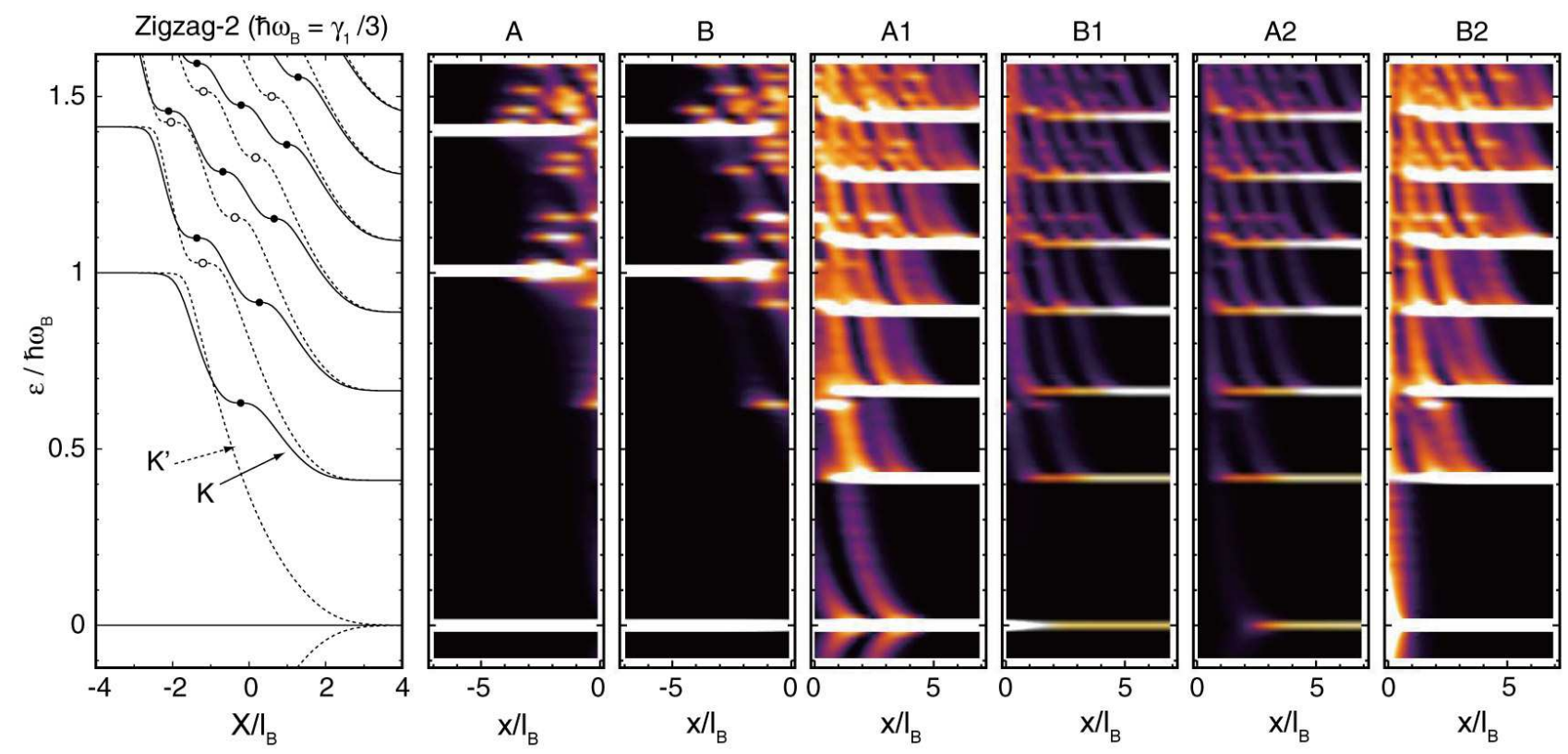

FIG. 9: (Right) Averaged local density of states at different atomic sites and (left) corresponding energy spectrum in boundary ZZ2 at magnetic field $\hbar \omega_{B}=\gamma_{1} / 3(B \sim 10 \mathrm{~T})$. The width of each bin in energy is taken as $0.02 \hbar \omega_{B}$.

Acknowledgments

This work was supported in part by Grant-in-Aid for Scientific Research on Priority Area "Carbon Nanotube Nanoelectronics," by Grant-in-Aid for Scientific Research, by Global Center of Excellence Program at Tokyo Tech "Nanoscience and Quantum Physics" from the Ministry of Education, Culture, Sports, Science and Technology, Japan, and by JST-EPSRC Japan-UK Cooperative Programme under Grant No. EP/H025804/1.

\section{Appendix A: Special flat-band points}

Here we prove that, at a special point $(\varepsilon, X)$ where a wavefunction satisfies Eq. (40), the derivative of Landau level in $X$ vanishes in $\mathrm{ZZ1}, \mathrm{ZZ2}$, and $\mathrm{AC}$, and the second derivative also vanishes in ZZ2. The condition Eq. (40) is alternatively written as

$$
\operatorname{det} M_{\mathrm{ZZ} 1}^{v}=\operatorname{det} M_{\mathrm{ZZ} 2}^{v}=0 \text {. }
$$

In the following, we will show that Eq. (A1) leads to

$$
\begin{aligned}
& \frac{\partial}{\partial X} \operatorname{det} M_{\mathrm{ZZ} 1}^{v}=\frac{\partial}{\partial X} \operatorname{det} M_{\mathrm{AC}}=0 \\
& \frac{\partial}{\partial X} \operatorname{det} M_{\mathrm{ZZ} 2}^{v}=\frac{\partial^{2}}{\partial X^{2}} \operatorname{det} M_{\mathrm{ZZ} 2}^{v}=0,
\end{aligned}
$$


which immediately proves the statements above.

In the matrices $M_{\mathrm{ZZ} 1}^{v}$ and $M_{\mathrm{ZZ} 2}^{v}$, we can eliminate $\phi_{\nu-1}^{L, R}$ by replacing them with $\phi_{\nu}^{L, R}$ and $\phi_{\nu+1}^{L, R}$, using the recursion formula of Weber's function,

$$
D_{\nu+1}-z D_{\nu}+\nu D_{\nu-1}=0 .
$$

Eq. (A1) at $v=K$ can then be transformed as

$$
\begin{aligned}
& \sum_{\mu= \pm} \mu\left(\varepsilon^{2}-\nu_{\bar{\mu}}\right) \phi_{\nu_{\bar{\mu}}}^{R}\left(\phi_{\nu}^{L} \phi_{\nu_{\mu}+1}^{R}+\phi_{\nu+1}^{L} \phi_{\nu_{\mu}}^{R}\right)=0 \\
& \sum_{\mu= \pm} \mu\left(\varepsilon^{2}-\nu_{\bar{\mu}}\right) \phi_{\nu_{\bar{\mu}+1}}^{R}\left(\phi_{\nu}^{L} \phi_{\nu_{\mu}+1}^{R}+\phi_{\nu+1}^{L} \phi_{\nu_{\mu}}^{R}\right)=0
\end{aligned}
$$

with $\bar{\mu}=-\mu$, leading to

$$
\phi_{\nu}^{L} \phi_{\nu_{\mu}+1}^{R}+\phi_{\nu+1}^{L} \phi_{\nu_{\mu}}^{R}=0,
$$

for both of $\mu= \pm$.

The derivative of the matrix determinant in $X$ can be evaluated using Eq. (10). For $M_{\mathrm{ZZ} 1}^{K}$, we obtain

$$
\begin{gathered}
\frac{\partial \operatorname{det} M_{\mathrm{ZZ} 1}^{K}}{\partial X} \propto \sum_{\mu= \pm} \mu\left(\varepsilon^{2}-\nu_{\bar{\mu}}\right) \phi_{\nu_{\bar{\mu}+1}}^{R \prime}\left(\phi_{\nu}^{L} \phi_{\nu_{\mu}+1}^{R}+\phi_{\nu+1}^{L} \phi_{\nu_{\mu}}^{R}\right) \\
\left.-\phi_{\nu}^{L} \sum_{\mu= \pm} \mu\left(\varepsilon^{2}-\nu_{\mu}\right)\left(\varepsilon^{2}-\nu_{\bar{\mu}}\right) \phi_{\nu_{\mu}}^{R} \phi_{\nu_{\bar{\mu}+1}}^{R}, \quad \text { (A8 }\right)
\end{gathered}
$$

where ' represents the derivative in $X$. At the points satisfying Eq. (A1), the first term becomes zero because of Eq. (A7). The second term is transformed with Eq. (A7) as

$$
\phi_{\nu+1}^{L} \sum_{\mu= \pm} \mu\left(\varepsilon^{2}-\nu_{\mu}\right)\left(\varepsilon^{2}-\nu_{\bar{\mu}}\right) \phi_{\nu_{\mu}}^{R} \phi_{\nu_{\bar{\mu}}}^{R},
$$

which vanishes since the argument inside the summation is anti-symmetric in $\mu$.

For $M_{\mathrm{ZZ} 2}^{K}$, we have

$$
\frac{\partial \operatorname{det} M_{\mathrm{ZZ} 2}^{K}}{\partial X} \propto \sum_{\mu= \pm} \mu\left(\varepsilon^{2}-\nu_{\bar{\mu}}\right) \phi_{\nu_{\bar{\mu}}}^{R \prime}\left(\phi_{\nu}^{L} \phi_{\nu_{\mu}+1}^{R}+\phi_{\nu+1}^{L} \phi_{\nu_{\mu}}^{R}\right),
$$

which similarly vanishes under the condition Eq. (A7). Eq. (A10) is even differentiated as

$$
\begin{gathered}
\frac{\partial^{2} \operatorname{det} M_{\mathrm{ZZ} 2}^{K}}{\partial X^{2}} \propto \sum_{\mu= \pm} \mu\left(\varepsilon^{2}-\nu_{\bar{\mu}}\right) \phi_{\nu_{\bar{\mu}}}^{R \prime \prime}\left(\phi_{\nu}^{L} \phi_{\nu_{\mu}+1}^{R}+\phi_{\nu+1}^{L} \phi_{\nu_{\mu}}^{R}\right) \\
-\phi_{\nu}^{L} \sum_{\mu= \pm} \mu\left(\varepsilon^{2}-\nu_{\mu}\right)\left(\varepsilon^{2}-\nu_{\bar{\mu}}\right) \phi_{\nu_{\mu}}^{R} \phi_{\nu_{\bar{\mu}}}^{R \prime} . \quad(\mathrm{A} 11)
\end{gathered}
$$

The first term becomes zero again under Eq. (A7). The factor $\left(\phi_{\nu_{\bar{\mu}}}^{R}\right)^{\prime}$ in the second term can be written in terms $\phi_{\nu_{\bar{\mu}}}^{R}$ and $\phi_{\nu_{\bar{\mu}+1}^{R}}^{R}$ using Eq. (10). Then it is shown to vanish by similar transformation to Eq. (A9).

The determinant of $M_{\mathrm{AC}}$ can be written in terms of those of ZZ1 and ZZ2 as,

$$
\operatorname{det} M_{\mathrm{AC}}=\operatorname{det} M_{\mathrm{ZZ} 1}^{K} \operatorname{det} M_{\mathrm{ZZ} 2}^{K^{\prime}}-\operatorname{det} M_{\mathrm{ZZ} 2}^{K} \operatorname{det} M_{\mathrm{ZZ} 1}^{K^{\prime}} \text {. }
$$

Under the condition $\operatorname{det} M_{\mathrm{ZZ} 1}^{K}=\operatorname{det} M_{\mathrm{ZZ} 2}^{K}=$ $\left(\operatorname{det} M_{\mathrm{ZZ} 1}^{K}\right)^{\prime}=\left(\operatorname{det} M_{\mathrm{ZZ} 2}^{K}\right)^{\prime}=0$, Eq. (A12) immediately gives $\left(\operatorname{det} M_{\mathrm{AC}}\right)^{\prime}=0$.

\section{Appendix B: Nearly-zero energy states}

Let us focus on the eigenstates in the vicinity of zero energy, taking the case of ZZ2 as an example. We will show here that the zero-energy levels in monolayerbilayer junction are contributed not only by the Landau levels in bulk monolayer and bilayer, but also by the zero-energy edge states localized to the boundary. For $K$ point, there are two independent wavefunctions exactly at zero energy,

$$
\Psi^{K 1}=\left\{\begin{array}{l}
\left(\begin{array}{l}
F_{A}^{K} \\
F_{B}^{K}
\end{array}\right)=\left(\begin{array}{c}
0 \\
\phi_{0}
\end{array}\right) \quad(x<0) ; \\
\left(\begin{array}{l}
F_{A 1}^{K} \\
F_{B 1}^{K} \\
F_{A 2}^{K} \\
F_{B 2}^{K}
\end{array}\right)=\left(\begin{array}{c}
0 \\
\phi_{0} / \tilde{\gamma}_{1} \\
0 \\
-i \phi_{1}
\end{array}\right) \quad(x>0),
\end{array}\right.
$$

and

$$
\Psi^{K 2}=\left\{\begin{array}{l}
\left(\begin{array}{c}
F_{A}^{K} \\
F_{B}^{K}
\end{array}\right)=\left(\begin{array}{c}
0 \\
0
\end{array}\right) \quad(x<0) ; \\
\left(\begin{array}{c}
F_{A 1}^{K} \\
F_{B 1}^{K} \\
F_{A 2}^{K} \\
F_{B 2}^{K}
\end{array}\right)=\left(\begin{array}{c}
0 \\
0 \\
0 \\
\phi_{0}
\end{array}\right) \quad(x>0),
\end{array}\right.
$$

with $\phi_{n} \equiv \phi_{n}^{R}=(-1)^{n} \phi_{n}^{L}$ for a nonnegative integer $n$, and the overall normalization factor is omitted.

In $X \rightarrow \infty$, i.e., when the center coordinate goes deep inside of the bilayer region, $\Psi^{K 1}$ and $\Psi^{K 2}$ approach the wavefunctions of two zero-energy Landau levels of bulk bilayer graphene. In $X \rightarrow-\infty$, on the other hand, $\Psi^{K 1}$ becomes the only zero-energy level of the bulk monolayer, while $\Psi^{K 2}$ does not have any amplitudes in the monolayer side, but mostly concentrated on $B_{2}$ sites near the boundary. $\Psi^{K 2}$ at $B_{2}$ then approximates

$$
\phi_{0} \propto e^{-(x-X)^{2} / l_{B}^{2}} \approx \text { const. } \times e^{-k_{y} x},
$$

which is independent of magnetic field. This corresponds to the zero-energy edge mode in zero magnetic field limit 53

For $K^{\prime}$-point, we have a single state at zero energy,

$$
\Psi^{K^{\prime} 1}=\left\{\begin{array}{l}
\left(\begin{array}{c}
F_{A}^{K} \\
F_{B}^{K}
\end{array}\right)=\left(\begin{array}{c}
\phi_{0} \\
0
\end{array}\right) \quad(x<0) ; \\
\left(\begin{array}{c}
F_{A 1}^{K} \\
F_{B 1}^{K} \\
F_{A 2}^{K} \\
F_{B 2}^{K}
\end{array}\right)=\left(\begin{array}{c}
\phi_{0} \\
0 \\
0 \\
0
\end{array}\right) \quad(x>0) .
\end{array}\right.
$$

When $X$ moves from $-\infty$ to $+\infty$, the wavefunction $\Psi^{K^{\prime} 1}$ crosses over from the only zero-energy level in monolayer to one of zero-energy levels in bilayer, $\nu_{-}=-1$. 
Besides, for positive large $X$, there exist another two levels near zero energy, which are expressed as a hybridization of bilayer's Landau level of $\nu_{+}=0$ and a zero-energy edge state. The derivation goes as follows. By expanding $\nu_{ \pm}$in Eq. (21) in $\varepsilon$, the determinant of $M_{\mathrm{ZZ} 2}^{K^{\prime}}$ can be written in a small $|\varepsilon|$ as,

$$
\operatorname{det} M_{\mathrm{ZZ} 2}^{K^{\prime}}=-\frac{\varepsilon}{\gamma_{1}} \phi_{\nu_{+}}^{R}\left(\phi_{\nu}^{L} \phi_{\nu_{-}}^{R}+\phi_{\nu_{-1}}^{L} \phi_{\nu_{-}+1}^{R}\right)+O\left(\varepsilon^{3}\right) .
$$

The energies of the nearly zero-energy states in question are given by the condition $\phi_{\nu_{+}}^{R}=0$, when Eq. (B5) vanishes. For a large $X$, the function $\phi_{\nu_{+}}^{R}$ can be evaluated by the asymptotic expansion of $D_{\nu}(z)$ which stands for large $|z|$ :

$$
\begin{aligned}
D_{\nu}(z) \sim\left\{\begin{array}{c}
D_{\nu}^{(1)}(z) \quad(|\arg z|<3 \pi / 4) \\
D_{\nu}^{(1)}(z)+e^{ \pm \nu \pi i} D_{\nu}^{(2)}(z) \\
(\pi / 4< \pm \arg z<5 \pi / 4)
\end{array}\right. \\
D_{\nu}^{(1)}(z)=e^{-z^{2} / 4} z^{\nu} \sum_{k=0}^{\infty}(-1)^{k} \frac{\nu(\nu-1) \cdots(\nu-2 k+1)}{k ! 2^{k} z^{2 k}} \\
D_{\nu}^{(2)}(z)=-\frac{\sqrt{2 \pi}}{\Gamma(-\nu)} e^{z^{2} / 4} z^{-\nu-1} \\
\quad \times \sum_{k=0}^{\infty} \frac{(\nu+1)(\nu+2) \cdots(\nu+2 k)}{k ! 2^{k} z^{2 k}}
\end{aligned}
$$

When $z$ is negative and $|\nu|$ is small, it approximates

$$
D_{\nu}(z) \approx e^{-z^{2} / 4}+\sqrt{2 \pi} \nu \frac{e^{z^{2} / 4}}{z} .
$$

This leads to an approximate expression $\phi_{\nu_{+}}^{R}(x=0)$ for positive large $X$,

$$
\phi_{\nu_{+}}^{R} \approx e^{-\left(X / l_{B}\right)^{2} / 2}-\sqrt{2 \pi} \nu_{+} \frac{e^{\left(X / l_{B}\right)^{2} / 2}}{\sqrt{2} X / l_{B}} .
$$

$\phi_{\nu_{+}}^{R}$ becomes zero at $\nu_{+}=\left(X / l_{B}\right) e^{-\left(X / l_{B}\right)^{2}} / \sqrt{\pi}$, giving the energies of nearly-zero energy mode,

$$
\varepsilon_{ \pm}^{K^{\prime} 2} \approx \pm \sqrt{\frac{X / l_{B}}{\sqrt{\pi}\left(1+\gamma_{1}^{2}\right)} e^{-\left(X / l_{B}\right)^{2}}}
$$

where we used $\nu_{+}(\varepsilon) \approx\left(1+\tilde{\gamma}_{1}^{2}\right) \varepsilon$ for small $\varepsilon$. The corresponding wavefunctions in the bilayer part are written as

$$
\begin{aligned}
& \Psi_{ \pm}^{K^{\prime} 2} \approx \Psi_{\text {bulk }}^{K^{\prime} 2} \pm \Psi_{\text {edge }}^{K^{\prime} 2} \\
& \Psi_{\text {bulk }}^{K^{\prime} 2}=\left(\begin{array}{c}
i \phi_{1+\nu_{+}} \\
0 \\
-\phi_{0+\nu_{+}} / \tilde{\gamma}_{1} \\
0
\end{array}\right), \\
& \Psi_{\text {edge }}^{K^{\prime} 2}=\left(\begin{array}{c}
|\tilde{\varepsilon}| \phi_{0+\nu_{+}} \\
0 \\
i\left(1+\gamma_{1}^{2}\right)\left(|\tilde{\varepsilon}| / \tilde{\gamma}_{1}\right) \phi_{-1+\nu_{+}}
\end{array}\right) .
\end{aligned}
$$

In $X \rightarrow \infty$, the energy $\varepsilon_{ \pm}^{K^{\prime} 2}$ becomes exponentially small and $\Psi_{\text {bulk }}^{K^{\prime} 2}$ coincides with the zero-energy Landau level of bilayer, $\nu_{+}=0$. For $\Psi_{\text {edge }}^{K^{\prime} 2}, B 2$ component is nearly proportional to $D_{-1}(z)$, and approximates $\propto e^{-k_{y} x}$ near $x=0$. This is a zero-energy edge state localized near the boundary in the bilayer region. ${ }^{53}$ Thus the states $\Psi_{ \pm}^{K^{\prime} 2}$ are described as a hybridization of the bulk bilayer Landau level and zero-energy edge states.
1 J. W. McClure, Phys. Rev. 104, 666 (1956).

2 J. C. Slonczewski and P. R. Weiss, Phys. Rev. 109, 272 (1958).

3 D. P. DiVincenzo and E. J. Mele, Phys. Rev. B 29, 1685 (1984).

4 N. H. Shon and T. Ando, J. Phys. Soc. Jpn. 67, 2421 (1998).

5 Y. Zheng and T. Ando, Phys. Rev. B 65, 245420 (2002).

6 V. P. Gusynin and S. G. Sharapov, Phys. Rev. Lett. 95, 146801 (2005).

7 N. M. R. Peres, F. Guinea, and A. H. Castro Neto, Phys. Rev. B 73, 125411 (2006).

8 T. Ando, J. Phys. Soc. Jpn. 74, 777 (2005).

9 E. McCann and V. I. Falko, Phys. Rev. Lett. 96, 086805 (2006).

10 M. Koshino and T. Ando, Phys. Rev. B 73, 245403 (2006).

11 M. I. Katsnelson, Euro. Phys. J. B 52, 151 (2006).

12 J. Nilsson, A. H. Castro Neto, N. M. R. Peres, and F.
Guinea, Phys. Rev. B 73, 214418 (2006).

13 F. Guinea, A. H. Castro Neto, and N. M. R. Peres, Phys. Rev. B 73, 245426 (2006).

14 E. McCann, Phys. Rev. B 74, 161403 (2006).

15 E. V. Castro, K. S. Novoselov, S. V. Morozov, N. M. R. Peres, J. M. B. Lopes dos Santos, J. Nilsson, F. Guinea, A. K. Geim, and A. H. Castro Neto, Phys. Rev. Lett. 99, 216802 (2007).

16 M. Koshino, New J. Phys. 11, 095010 (2009).

17 K. S. Novoselov, A. K. Geim, S. V. Morozov, D. Jiang, Y. Zhang, S. V. Dubonos, I. V. Grigorieva, and A. A. Firsov, Science 306, 666 (2004).

18 K. S. Novoselov, E. McCann, S. V. Morozov, V. I. Falko, M. I. Katsnelson, U. Zeitler, D. Jiang, F. Schedin, and A. K. Geim, Nature Phys. 2, 177 (2006).

19 C. Berger, Z. Song, T. Li, X. Li, A. Y. Ogbazghi, R. Feng, Z. Dai, A. N. Marchenkov, E. H. Conrad, P. N. First, and W. A. de Heer, J. Phys. Chem. B 108, 19912 (2004). 
20 T. Ohta, A. Bostwick, T. Seyller, K. Horn, and E. Rotenberg, Science 313, 951 (2006)

${ }^{21}$ K. S. Novoselov, A. K. Geim, S. V. Morozov, D. Jiang, M. I. Katsnelson, I. V. Grigorieva, S. V. Dubonos, and A. A. Firsov, Nature 438, 197 (2005).

${ }^{22}$ Y. Zhang, Y.-W. Tan, H. L. Stormer, and P. Kim, Nature 438, 201 (2005).

23 M. Koshino and T. Ando, Physica E 40, 1014 (2008).

24 M. Koshino and E. McCann, Phys. Rev. B 81, 115315 (2010).

25 M. Fujita, K. Wakabayashi, K. Nakada. and K. Kusakabe, J. Phys. Soc. Jpn. 65, 1920 (1996).

${ }^{26}$ K. Nakada, M. Fujita, G. Dresselhaus, and M. S. Dresselhaus, Phys. Rev. B 54, 17954 (1996).

27 K. Wakabayashi, Phys. Rev. B 64, 125428 (2001).

${ }^{28}$ E. McCann and V. I. Falko, J. Phys.: Condens. Matter 16, 2371 (2004).

${ }^{29}$ L. Brey and H. A. Fertig, Phys. Rev. B 73, 235411 (2006).

30 N. M. R. Peres, A. H. Castro Neto, and F. Guinea, Phys. Rev. B 73, 241403 (2006).

31 K. Wakabayashi, J. Phys. Soc. Jpn. 71, 2500 (2002).

${ }^{32}$ K. Wakabayashi, M. Fujita, H. Ajiki, and M. Sigrist, Phys. Rev. B 59, 8271 (1999).

33 K. Wakabayashi and M. Sigrist, Phys. Rev. Lett. 84, 3390 (2000).

34 Y.-W. Son, M. L. Cohen, and S. G. Louie, Phys. Rev. Lett. 97, 216803 (2006).

35 Y.-W. Son, M. L. Cohen, and S. G. Louie, Nature 444, 347 (2006).

36 B. Obradovic, R. Kotlyar, F. Heinz, P. Matagne, T. Rakshit, M. D. Giles, M. A. Stettler, and D. E. Nikonov, Appl. Phys. Lett. 88, 142102 (2006).

37 L. Yang, C.-H. Park, Y.-W. Son, M. L. Cohen, and S. G. Louie, Phys. Rev. Lett. 99, 186801 (2007).

38 L. Yang, M. L. Cohen, and S. G. Louie, Phys. Rev. Lett. 101, 186401 (2008).

39 T. C. Li and S.-P. Lu, Phys. Rev. B 77, 085408 (2008).

40 H. Raza and E. C. Kan, Phys. Rev. B 77, 245434 (2008).

41 V. Ryzhii, M. Ryzhii, A. Satou, and T. Otsuji, J. Appl. Phys. 103, 094510 (2008).
42 T. Wassmann, A. P. Seitsonen, A. M. Saitta, M. Lazzeri, and F. Mauri, Phys. Rev. Lett. 101, 096402 (2008).

43 A. R. Akhmerov and C. W. J. Beenakker, Phys. Rev. B 77, 085423 (2008).

${ }^{44}$ V. H. Nguyen, V. N. Do, A. Bourne, V. L. Nguyen, and P. Dollfus, J. Phys.: Conf. Ser. 193, 012100 (2009).

45 D. Gunlycke and C. T. White, Phys. Rev. B 81, 075434 (2010).

46 B. Sahu, H. Min, A. H. MacDonald, and S. K. Banerjee, Phys. Rev. B 78, 045404 (2008).

47 E. V. Castro, N. M. R. Peres, J. M. B. Lopes dos Santos, A. H. Castro Neto, and F. Guinea, Phys. Rev. Lett. 100, 026802 (2008).

48 D. A. Abanin, P. A. Lee, and L. S. Levitov, Phys. Rev. Lett. 96, 176803 (2006).

49 D. A. Abanin, K. S. Novoselov, U. Zeitler, P. A. Lee, A. K. Geim, and L. S. Levitov, Phys. Rev. Lett. 98, 196806 (2007).

50 D. A. Abanin, P. A. Lee, and L. S. Levitov, Solid State Commun. 143, 77 (2007).

51 J. Nilsson, A. H. Castro Neto, F. Guinea, and N. M. R. Peres, Phys. Rev. B 76, 165416 (2007).

52 J. W. Gonzalez, H. Santos, M. Pacheco, L. Chico, and L. Brey, Phys. Rev. B 81, 195406 (2010).

53 T. Nakanishi, M. Koshino, and T. Ando, arXiv:1008.4450v1, to be published in Phys. Rev. B.

54 A. Misu, E. Mendez, and M. S. Dresselhaus, J. Phys. Soc. Jpn. 47, 199 (1979).

55 T. Nakanishi and T. Ando, J. Phys. Soc. Jpn. 77, 024703 (2008).

56 Y. Kobayashi, K. Fukui, T. Enoki, K. Kusakabe, and Y. Kaburagi, Phys. Rev. B 71, 193406 (2005).

57 Y. Niimi, T. Matsui, H. Kambara, K. Tagami, M. Tsukada, and H. Fukuyama, Phys. Rev. B 73, 085421 (2006).

58 T. Matsui, H. Kambara, Y. Niimi, K. Tagami, M. Tsukada, and H. Fukuyama, Phys. Rev. Lett. 94, 226403 (2005).

${ }^{59}$ Y. Niimi, H. Kambara, T. Matsui, D. Yoshioka, and H. Fukuyama, Phys. Rev. Lett. 97, 236804 (2006). 